\title{
Development of Exercise Intensity Monitoring System Based on Wearable Device
}

\author{
Yurong Chen ${ }^{1}$ Ran $_{\mathrm{Yi}^{2}}{ }^{2}$ \\ ${ }^{1}$ Hainan medical university, Hainan haikou 571199, China \\ ${ }^{2}$ Cnooc HaiNan Gas Co., LTD, Hainan Haikou 578001, China \\ paperiset@163.com
}

Keywords: Exercise Intensity, Monitoring System, Wearable Device, Accelerometer

\begin{abstract}
In this paper, we study heart rate, metabolic equivalent, the degree of subjective sensation and motion energy consumption characteristics. From the basic response characteristics of heart rate of sports load Heart rate measurement using the target heart rate to arrange exercise load strength and heart rate measurement, the characteristics of the intensity of heart rate monitoring analysis of the characteristics of heart rate index. We then design a real-time, wearable and motion artifact reduced health monitoring device. The wearable monitoring device, is consists of photoplethysmography sensor, 3-axis accelerometer, microprocessor and wireless module. The sensor acquire distorted heart beat signal due to motion. The test results show that the system runs normally and safely. The system satisfies the needs of monitoring exercise intensity and promoting persons to do moderate exercise.
\end{abstract}

\section{Introduction}

Sports can promote health, improve cardiopulmonary function, increase bone density and muscle strength, improve or eliminate negative emotions, improve the reaction ability, appropriate exercise helps the body health, excessive exercise is easy to damage the body health evaluation exercise intensity, is the important index used to describe the physical exercise on human physiological stimulation.

Current intensity monitoring indicators are mainly physical load intensity, intensity of physiological load intensity and subjective feeling, motion in a different way, the indicators are also different, should according to the application conditions and scope, using different indicators, such as physiological load intensity index monitoring intensity should be considered when the joint application of practical application several indicators, and operate simple aerobic exercise intensity monitoring indicators have heart rate metabolic Equivalent (Metablic Equivalent, MET) subjective feeling (RPE) movement energy expenditure, etc Heart rate refers to the number of heart beats per minute, can reflect the various physiological changes in the situation, to accurately describe the body movement to stimulate the immediate reaction and chronic adaptation, when adult quiet heart rate between $60 \sim 100$ times/min, an average of 75 times/min.Metabolic equivalent (metablic equivalent, MET) is relative to the important parameters of energy metabolism and the exercise intensity to sit a energy consumption as the basic reference, expounds the motion relative to the level of energy metabolism indexes, $1 \mathrm{MET}=$ oxygen consumption $3.5 \mathrm{~mL} /(\mathrm{kg} / \mathrm{min})$, applicable to assess cardiopulmonary function;Subjective feeling (RPE) using subjective evaluation exercise intensity, can be divided into the quiet very easy very relax a little strenuous effort is very laborious very laborious, corresponds to the heart rate increases in turn;Movement energy expenditure described within the allotted time (for example, $60 \mathrm{~min}$ ) the heat energy consumed by the form of different sports.

We design a real-time, wearable and motion artifact reduced health monitoring device. A wearable health monitoring device, is consists of sensor, 3-axis accelerometer, microprocessor and wireless module. The sensor acquire distorted heart beat signal due to motion. The test results show that the system runs normally and safely. The system satisfies the needs of monitoring exercise intensity and promoting persons to do moderate exercise. 


\section{Our Approach}

Hardware Description. The wearable sensor device should be small and light and attach to body tightly to reduce noise effect and feel comfortable to wear. Additionally, wireless communication device also reduces motion artifact by reducing effect of the data cable inertia. The sensor is attached to the body base, as shown in Figure 1.

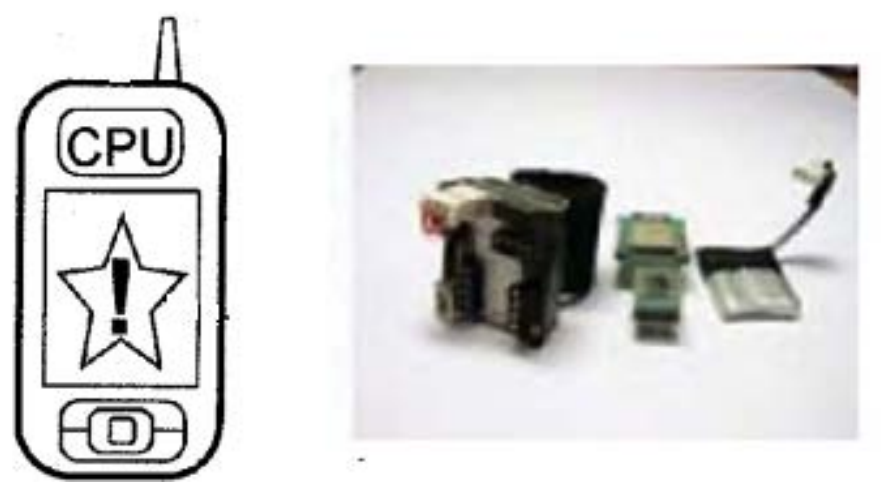

Figure 1. Hardware design for the sensor.

Processing Flow. The size and weight is $25 \times 30 \mathrm{~mm} 2$ and $16.8 \mathrm{~g}$, respectively. The sensor is located on the inner layer of the band, and the accelerometer is on the circuit. The band device is divided into two parts; the sensor device and the host analysis computer. In detail, the sensor device part can be divided into three parts, as shown in Figure 2.

The first sensor part consists of a light source, a photo sensor for PPG and an accelerometer for motion detection. A $940 \mathrm{~nm}$ wavelength, a surface mounted type Infrared LED, and a photo-diode are used to acquire the PPG signal. Although a more detailed explanation follow in the next chapter, motion is considered to be directly related to noise, so to measure and analysis the body motion and a noise source, a 3-axis accelerometer is used. Next part is the pre-signal processing, circuit part, which contains an analog signal processing part, amplifiers, filters, and analog to digital converters (ADC). Since the raw signal on the sensor signal (especially the AC component) is so small and distorted, signal processing is demanded before being sent to a communication system. The raw signal demands a low pass filter for reducing high frequency noise and high pass filter for rejecting a DC component to enhance the AC component. As filters, second order active analog high and low pass filters (Sallen-Key Filter) are used. Filtering signals are amplified to enhance and acquire discriminable signals by a thousand times. The last part is digital signal processing. High order filtering has good performance to extract wanted signal, but more number of components are required to increase filtering order.

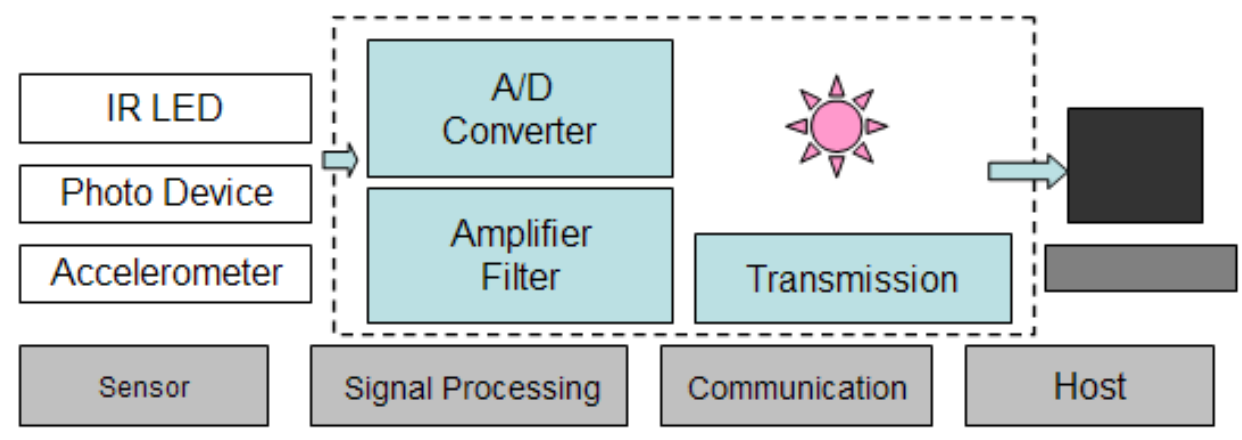

Figure 2. Processing flow of the sensor.

Therefore, digital filtering is employed to satisfy both circuit size and filtering performance. The filters are designed as a $0.5-3 \mathrm{~Hz}$ band pass filter, and totally fourth order analog active filter and digital filter are used in this signal processing. The following part is the communication system. To transmit obtained data from the sensor device to the host computer, the microcontroller converts data into communication language, an 8bit digital signal. Then it transmits the data through a wireless 
device, Bluetooth. It is a widely used device, especially for portable devices such as in MP3 players or cellular phones. The device has up to $30 \mathrm{~m}$ communication distance, and size of the device is $20 \mathrm{x}$ $18 \mathrm{~mm} 2$. The operation voltage is $3.7 \mathrm{~V}$. Labview software from National Instrument obtains transmitted data and display by graph on the host computer. Also, digital filtering and analysis algorithm are programmed.

\section{Equipment software implementation}

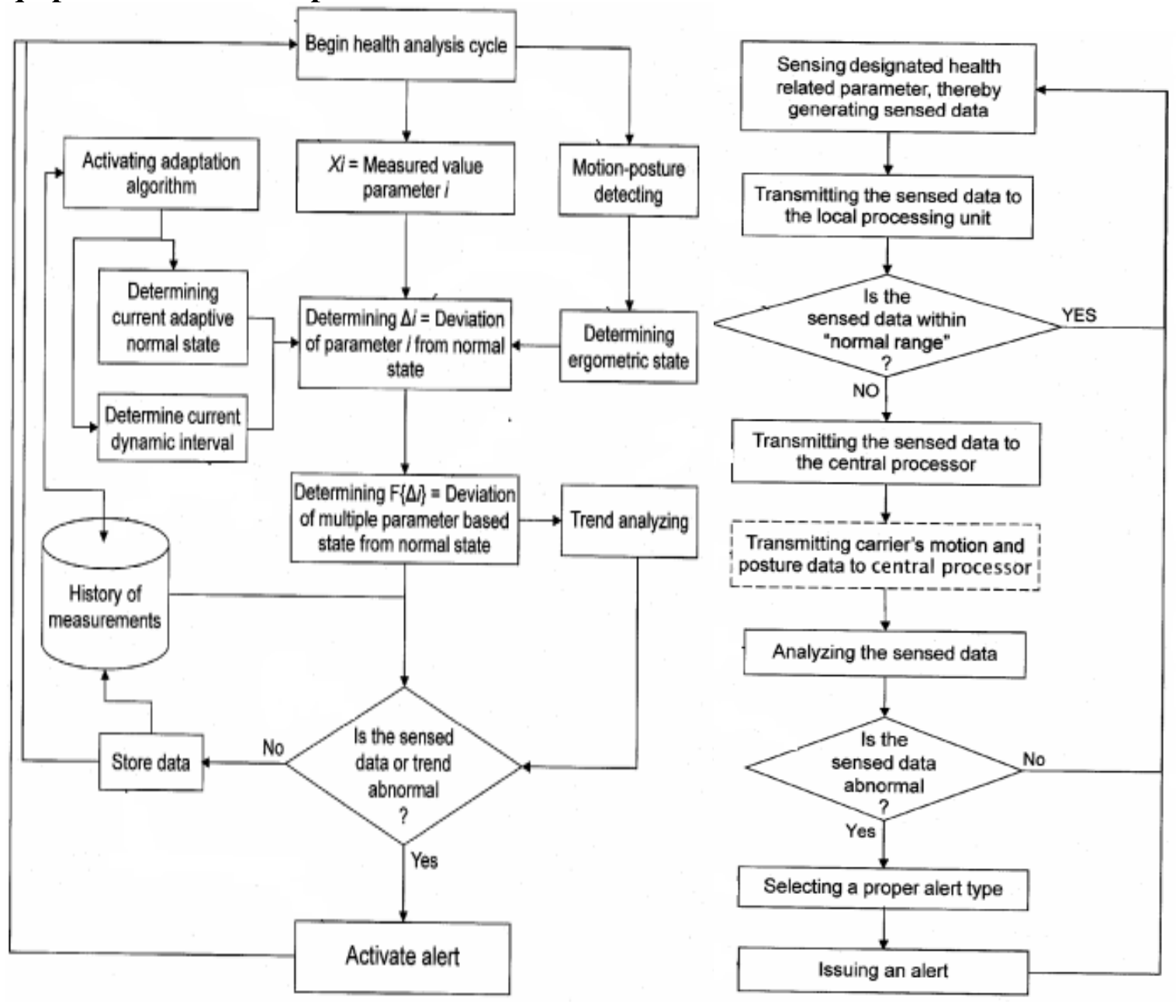

Figure 3. Processing flow of data acquisition.

Equipment acquisition of the software part mainly realize the $3 \mathrm{~d}$ acceleration sensor data collection, processing, storage and sending, as well as to the battery monitoring program, but are often low cost single chip microcomputer data processing ability is limited, the data storage capacity is small, but also have to meet the requirements of low power consumption equipment, so the software design procedure optimization, makes the power consumption to a minimum. The equipment acquisition software mainly based on TI the BLE protocol stack of development, the core architecture consists of two parts, the OSAL and HAL, based mainly on news events, tasks, and to implement the relevant business.

\section{Experiments and Results}

In order to verify the accuracy of the information this topic to human activity, we in 12 healthy adult subjects ( 6 men and 6 women, weight $45 \mathrm{~kg}$ to $90 \mathrm{~kg}$, aged between 15 and 45 years old), let them carry energy equipment for installing the software movement of real-time detection experiment. We ask that each tester wear the phone fixed in their waist, and start the program and timing, and record each tester under the variety of oxygen consumption and exercise data. In every three minutes to adjust the treadmill slope way to adjust the movement intensity is increased by $2 \%$, the running 
machine began to $2.7 \mathrm{~km} / \mathrm{h}, 10 \%$ grade, and with the increase of the slope in the process of exercise intensity increases, until the participants feel exhausted For male subjects, about 12 minutes. For female participants, about $9 \sim 10$ minutes During this period by mobile phone testing exercise and exercise treadmill measurements as shown in Table 1 and Table 2.

Table 1. Frequency of sport movement

\begin{tabular}{|c|c|c|c|}
\hline Motion & \multicolumn{3}{|c|}{ Frequency (Hz) } \\
\hline Run & \multicolumn{3}{|c|}{4} \\
\hline Walk & \multicolumn{3}{|c|}{3} \\
\hline Hand gesture & \multicolumn{3}{|c|}{$2-6$} \\
\hline Exploration & \multicolumn{3}{|c|}{$0.5-2.0$} \\
\hline Texture scan & \multicolumn{3}{|c|}{$0.9-2.8$} \\
\hline Hand writing & \multicolumn{3}{|c|}{$4-7$} \\
\hline Typing & \multicolumn{3}{|c|}{$5-9$} \\
\hline Tapping & \multicolumn{3}{|c|}{$6-9$} \\
\hline \multicolumn{4}{|c|}{ Table 2. Error signals on frequency conditions } \\
\hline Signal & \multicolumn{3}{|c|}{ Error signal } \\
\hline Comparison & $2.5(\mathrm{~Hz})$ & $3.0(\mathrm{~Hz})$ & $3.5(\mathrm{~Hz})$ \\
\hline Measurement & 27.00 & 84.36 & 92.31 \\
\hline Reconstructed & 3.12 & 3.10 & 4.26 \\
\hline
\end{tabular}

The results show experimental signals; lower periodical signal is reference pulsation signal, middle complex signal is measured corrupted signal, and top smooth and periodical signal is reconstructed signal. The counting error of pulsation signal is reduced less than $5 \%$. The experimental frequency condition is similar to a hand's daily movement. For example as hand motions, running, walking, and hand gesture have approximately 2 to $6 \mathrm{~Hz}$ frequency motion, and as finger motions, object exploration and texture scanning have 0.9 to $2.8 \mathrm{~Hz}$.

\section{Conclusion}

In this paper, a real-time, wearable and wireless finger band sensor is designed with the motion artifact reduction algorithms. We obtain body motion data which is source of motion artifact using the accelerometer, and it is applied to active noise cancellation algorithm. As experiments, one directional hand motions which has difference frequency conditions are given. Therefore, wearable and portable health care device with motion artifact reduced algorithm techniques are demanded for continuous health monitoring. And the proposed device in this paper could have a potential to developing this portable and wearable device without daily motion artifact.

\section{References}

[1] Godfrey A. Bourke AK, Olaighin GM, Van de Ven P, Nelson J. Activity classification using a single chest mounted tri-axial accelerometer. Medical Engineering \& Physics, 2011, 33(9): 1127-1135.

[2] Hong YJ, Ig-Jae K, Sang CA, Hyoung-Gon K. Mobile health monitoring system based on activity recognition using accelerometer. Simulation Modelling Practice and Theory, 2010, 4(18): 446-455.

[3] Field T. Exercise research on children and adolescents. Complementary Therapies in Clinical Practice, 2012, 1(18): 54- 59.

[4] Marcos S, Nadia A, Joshua S, et al. Fernando Naclerio Acute effects of a multinutrient supplement on performance, rate of perceived exertion and markers of catabolism in young resistance trainers practitioners. Journal of the International Society of Sports Nutrition, 2014, 12(11) : 9 . 
[5] Fernandez, Sanz M, et al. A comparison of the activity profile and physiological demands between advanced and recreational veteran tennis players. J Strength Condres, 2009, 23: 604610. 